\title{
NUMERICAL MODELLING OF HUMAN LIGAMENTS
}

\author{
Ferenc Szakály ${ }^{1}$, Imre Bojtár ${ }^{1}$, Gábor Szebényi ${ }^{2}$ \\ ${ }^{1}$ Department of Structural Mechanics, Budapest University of Technology and Economics \\ ${ }^{2}$ Department of Polymer Engineering, Budapest University of Technology and Economics \\ szakalyf@eik.bme.hu \\ DOI: 10.17489/biohun/2016/1/04
}

\begin{abstract}
During the everyday life, our articulations are subjected to numerous different static and dynamic effects, in the structure of the human body they ensuring the proper attachment between the articulating bones. Within them, the various ligaments take care of the stability of the joints, their healthy functioning is essential even for ordinary daily activities. Due to the extremely serious consequences of their damage or rupture, medical science has aimed to find an adequate way to heal or replace them with natural or artificial tendons/ligaments, but so far (mostly the long term) results are not satisfactory at all. For the improvement of these procedures the better understanding and description of their mechanical behaviour is necessary. Our articulations (knee, ankle, hand and shoulder) show very difficult mechanical behaviour both from the point of loading conditions and material behaviour. Thus, the trustworthy numerical simulation of them is a very complex problem, on the top of the irregular geometry, both geometrical and material nonlinearities have to be taken into account. That is why real laboratory experiments are indispensable for the reliable numerical results. For this purpose, series of experiments have been performed on tendons and ligaments in the Biomechanical Laboratory of the BME. By the evaluation of the results different hyperelastic material models are parameterized and compared with the help of simple finite element models. On the basis of these models a three-dimensional numerical model is developed in Ansys, validated by real and numerical results. On this model, frequently occurring motions are investigated, focusing on the mechanical response of the major knee joints..
\end{abstract}

Keywords: human ligaments, hyperelastic material models, laboratory experiments, finite element analysis

\section{Introduction}

In the human body, the knee joint is the largest and most complicated articulation, furthermore it owns the highest incidence of ligament injury, thus in this work this articulation had been studied. According to its mechanism it is a troche-ginglymus, ${ }^{1}$ which means that it can make a flexion-extension motion and a rotation about the axis of the shin. The motion of this articulation is a three-dimensional, continuously changing polycentric rotation, around permanently varying axes. It connects the femur and tibia with the help of the four major ligaments (lateral/medial collateral ligaments and anterior/posterior cruciate ligaments), which consist of strong, tough bands of tissue, and play the most serious role in the stability of the knee joint. They accomplish this stabilizing effect in such a way that they allow joints to move easily within normal limits and exert an increasing resistance against motions which are out of this normal range. From its complexity it follows that the mechanical behaviour is rather difficult to describe, it shows both anisotropic, inhomogeneous and nonlinear elastic/viscoelastic behaviour supplemented by three-dimensional compound loading 
conditions. Due to these facts, this joint is not completely revealed yet, there are lots of unexplored areas.

In the modelling of soft tissues like tendons and ligaments the constitutive relationships are the most significant and sensitive part of the problem. Actually, the ligament is a biological composite, composed of a ground substance which is reinforced with collagen and elastin (connective soft tissues). ${ }^{3}$ Collagen is the main load-bearing component of the ligaments ${ }^{4}$ while elastin ensures the elasticity of the tissues, therefore larger elastin content leads to higher ultimate strain. The main function of the ground substance is to hold together the soft tissues. The structure of a tendon or ligament can be seen on Figure 1.

The ligament splits into smaller units, called fascicles, which contain the basic fibres and fibroblasts. At this level it is very important from mechanical point of view that these fibres have a wavy pattern (crimp pattern) in the unloaded configuration. This specific pattern significantly contributes to the nonlinear behaviour of the ligaments in case of low stresses. The fibres further split into fibrils, subfibrils, microfibrils, and finally into collagen fibres.

Due to the specific structure of these soft tissues, during quasi-static loading we can distin- guish three different characteristic regions. ${ }^{3}$ In the unloaded configuration the collagen fibres follow a crimp pattern, this state is called slack configuration. The first region is the toe region, where the originally wavy collagen fibres are starting to gradually elongate and taking part in the load-bearing procedure. Since the strength and stiffness of the ligaments are ensured by the fibres, this region is (hardening) nonlinear (the ligaments are typically in this region when they only subjected to the everyday activities). During the straightening, theses fibres interact with the hydrated matrix, which means that they are also subjected to a pressure due to this process. As the load increases, in the linear region the collagen fibres straighten out, and the stress-strain curve becomes practically linear. In the slack configuration, the orientation of the fibres is irregular, but as they gradually elongating they become parallel with the applied load (and also with each other). As a consequence of this change in the orientation, after the straightening the ligaments can be considered as transversely isotropic instead of anisotropic. In the last one, namely the failure region, at higher stresses the fibres sequentially rupture (the stiffness of the ligament is continuously reducing) until the tear of the entire ligament. Besides the complex quasi-static behaviour tendons and ligaments show signif-

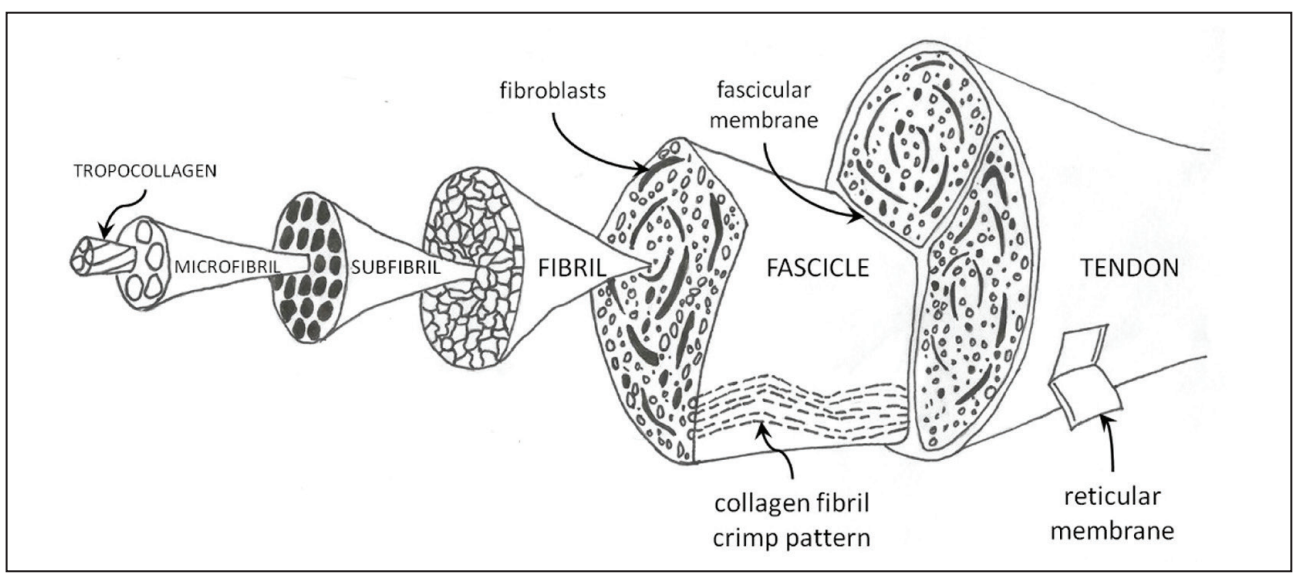

Figure 1. Structural hierarchy of ligament ${ }^{2}$ 
icant time-and history dependent viscoelastic behaviour, which involves creep and relaxation, strain rate dependence and hysteresis. The ligament injures usually happen at higher strain rates, thus the strain rate is an important parameter. The experiments show that for higher strain rates the ligaments behave stiffer, or in other words the slope of the stress-strain curve increases. On the other hand, strain rate has also an important role if we consider the failure mode for the bone-ligament-bone complexes, due to the high strain rate sensitivity of bones. In case of these structures at low strain rates the bony avulsion failure is the typical, but at higher ones the bone gets stronger and the failure of the ligament is the typical. With the help of laboratory experiments, the phenomena of creep and relaxation were observed firstly. ${ }^{5}$ These studies pointed out that the rate of creep is stress dependent, and the rate of relaxation is strain dependent, furthermore the relaxation proceeds faster than the creep, so the ligaments have a nonlinear viscoelastic behaviour. During the loading and unloading process at constant strain rate hysteresis loops can be observed at the stress-strain curves due to the dissipation of internal energy, they are translated to the right direction with the increasing loading cycles. ${ }^{6}$ Laboratory tests pointed out that the hysteresis decreases with the increasing number of loading-unloading cycles, and after sufficient number of repetition the mechanical response become repeatable. The concomitant of this process is an increasing in the length of the specimen. The precondition of the soft tissue has also positive side effects, namely it reduces the relaxation and creep, and decreases the peak stress during cyclic loading. As a consequence of this phenomena, the precondition of the ligaments is usually applied before acquiring the required material parameters by the laboratory experiments.

Because of the complex behaviour of ligaments, material models are also difficult to create, depending on the level of neglects and assumptions (e.g. failure region, viscous behaviour, anisotropy, etc.), there are lots of models exist. The most common material models from the family of phenomenological models are the hyperelastic ones (Neo-Hooke model, Mooney-Rivlin model) with large strains, usually their material parameters are determined by laboratory experiments. The majority of the models are one-dimensional, or isotropic three-dimensional, furthermore, due to the precondition the viscoelastic properties are also neglected. Over the last few decades, the researchers worked out several microstructural material models, in many times with very different approaches. Firstly, they concentrated to describe the toe region properly (with many different basic ideas) with elastic and viscoelastic models, and in the recent past the failure behaviour has become also the target of the scientists.

In the current numerical models both the modelling of a single ligament and the modelling of the whole joint with the connecting bones can be found, depending on the aim of the research. Due to the fact that the anterior cruciate ligament (ACL) is the most critical ligament from medical point of view, the majority of the researchers focusing on this one, but collateral ligament (MCL and LCL) models also can be found. In these three-dimensional models the accuracy is very important, but the geometry is highly complex, therefore in most cases they are based on laser scanning and medical imaging (CT, MRI). For example to the modelling of a single ligament both Haghpanahi et al. $^{5}$ and Feng Xie et al. investigated the stress distribution in the human ACL. The geometry was obtained by MRI images and laser scanner, and hyperelastic models were used at the incompressible, isotropic model of ligament. The aim of this simulation was to analyze the stress-distribution coming from the different load cases, and compare its characteristics with 
the available experimental data. Modelling of the complex joint can be applied more beneficially in case of the investigation of specific knee motions controlled by the bones. Untaroiu et al. $^{8}$ studied the femur-MCL-tibia complex with hyperelastic and hyper-viscoelastic (QLV theory was considered) material models for the quasi-static and dynamic simulations. J.A. Weiss with J.C. Gardiner ${ }^{9}$ also investigated the bony MCL complex during valgus knee motion, including the effect of in situ strains. Zhong Yan-lin et al. ${ }^{10}$ studied the lateral collateral ligament on 3D models at different knee flexion angles obtained by CT scans. We can note that the majority of the studies neglects the time-dependent behaviour, and focus on the stress distribution in the ligaments due to different knee joint motions.

\section{Methods and results}

2.1 Evaluation of the laboratory experiments, the applied material models

In order to use proper hyperelastic material models at the finite element modelling of the knee joint, laboratory tests were performed to parameterize different material models. The purpose of these experiments is to investigate such tendons in the leg, which are able to substitute the knee ligaments, mostly from medical point of view. The tendons were cut from human cadavers, after the death but within 24 hours, and then the physicians took care of the proper conservation till the experiments. Before the actual tests, the samples are gripped between the clamps, which leads to a frictionless, fixed support. After the precondition the tensile tests were performed applying quasi-static loading circumstances. The following series of loading phases (Figure 2) reflects very well the large deformation capability of tendons and ligaments, in some cases the samples become two or three times longer comparing to their original size.

The testing machine records the value of the load and elvongation ten times in a sec, thus our direct results are the force-displacement diagrams. In this work only two measurement results can be used to the material model (right
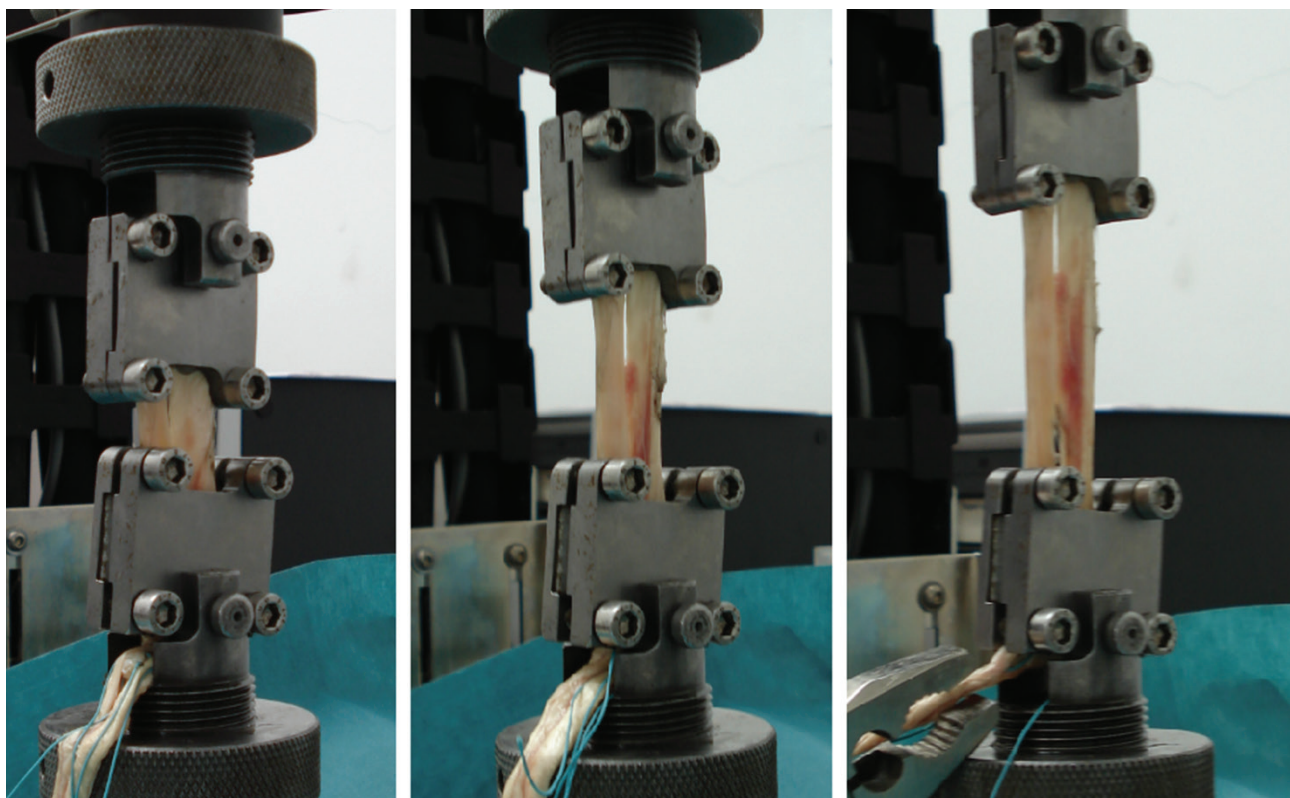

Figure 2. The different loading phases of the specimen 
Quadriceps tendon), but in case of more available data the evaluation process is exactly the same. Because of the large deviation, it is important to see the force-elongation diagrams of the samples (Figure 3), there is about $60 \%$ difference in the maximum load.

It is very important that in case of biological materials, in contrary the fact that both samples are the same type of tendons, the material behaviour varies with the age, sex, injury, treatment and experimental technique, which can lead to large variance. For the material parameters the Cauchy stress-strain relationships have to be obtained from F-e diagrams. The width, thickness and the length between the clamps were measured on the spot, thus the volume of the samples is given in this way. The effect of large strains have to be taken into account at these materials, the area of the cross section is continuously changing with the elongation. For this purpose, instead of using the initial area of the tendons to calculate the stresses, at every time-force-elongation pair the current area must be calculated. Utilizing the incompressibility assumption, the volume of the samples (theoretically) is not changing, hence the momentary areas can be determined by dividing the volume by the current length as well as the Cauchy stresses.

The aim of the experiments is to determine material parameters for hyperelastic material models, in this study for the Neo-Hooke, the 2, 3, 5 and 9 parameters Mooney Rivlin models. Once the stress-strain relationship is obtained for the 9 parameters version, all the others can be derived from it by eliminating the proper terms. The general form of the strain energy function in the 9 parameters case (1): ${ }^{11}$
The above expression is the functions of the invariants $\left(I_{p}, I_{2}\right)$ of the right Cauchy-Green deformation tensor, which can be easily expressed with the deformation-gradient tensor in the direction of the principle stretches. The $c$ constants are the parameters to be determined and $d$ constant is the incompressibility parameter. By taking into account the incompressibility assumption and the uniaxial loading condition according to the experiment the Cauchy stresses can be determined from the following formula (2):

$$
\sigma=\lambda \frac{\partial W\left(I_{1}^{\prime}, I_{2}^{\prime}\right)}{\partial \lambda}=\lambda\left(\frac{\partial W}{\partial I_{1}^{\prime}} \frac{\partial I_{1}^{\prime}}{\partial \lambda}+\frac{\partial W}{\partial I_{2}^{\prime}} \frac{\partial I_{2}^{\prime}}{\partial \lambda}\right)
$$

After the calculation of the necessary derivatives and some simplifications Cauchy stressstretch relationship can be obtained in the

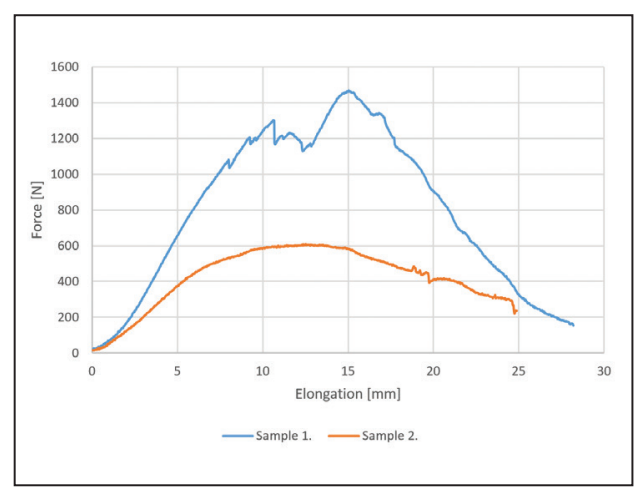

Figure 3. Force-elongation diagrams of the Quadriceps tendons

function of the unknown constants for the 9 parameters case, and after the adequate eliminations for all the other versions. Before the determination of the parameters, from the available stress-strain diagrams we should

$$
\begin{aligned}
W=c_{1}\left(\overline{I_{1}}-3\right) & +c_{2}\left(\overline{I_{2}}-3\right)+c_{3}\left(\bar{I}_{1}-3\right)^{2}+c_{4}\left(\bar{I}_{1}-3\right)\left(\bar{I}_{2}-3\right)+c_{5}\left(\bar{I}_{2}-3\right)^{2} \\
& +c_{6}\left(\bar{I}_{1}-3\right)^{3}+c_{7}\left(\bar{I}_{1}-3\right)^{2}\left(\bar{I}_{2}-3\right)+c_{8}\left(\bar{I}_{1}-3\right)\left(\bar{I}_{2}-3\right)^{2}+c_{9}\left(\bar{I}_{2}-3\right)^{3} \\
& +\frac{1}{d}(J-1)^{2}
\end{aligned}
$$


make an "average" diagram. Usually the measured curves are weighted with specific factors according to the reliability of the individual results and the exactness of the experiments, but in this case, when there are only two curves to make an average, we do not have enough information about the weighting aspects. Besides the averaging, the stress-strain diagrams should be examined at a reduced strain region, which is closer to the reality in physiological sense, namely in this work this limit is set to 0.4. The determination of the parameters is practically an optimization problem, where we have an approximation function $(\sigma)$ with some parameters $\left(c_{p}, c_{2}, . ., c_{n}\right)$, and the purpose is to minimize the deviation between the calculated function and the experimental curve by the optimal choice of the material constants. The mathematical form of the problem (3):

$$
\begin{gathered}
f\left(c_{1}, c_{2}, \ldots, c_{n}\right)=\sigma\left(c_{1}, c_{2}, \ldots, c_{n}\right)-\sigma_{\text {experimental }} \rightarrow \min ! \\
c_{1}+c_{2} \geq 0
\end{gathered}
$$

In this formula, $f$ is the function to be minimized, $\sigma$ is the Cauchy stress-stretch function and $\sigma_{\text {experimental }}$ represents the measured Cauchy stress values. The inequality condition has mechanical importance, it ensures the nonnegative starting slope of the function. There are different ways to solve this problem, in this study the nonlinear least squares method is utilized with the help of the Matlab R2013a mathematical software. After this procedure, the coefficients of determination $(C o D)$ are calculated in order to characterize the exactness of the approximation functions. The general formula of this index-number. After the determination of the material parameters and $C o D$ values for all the hyperelastic models, it can be concluded that Neo-Hooke model is not able to model properly soft tissues like tendons and ligaments, the coincidence of the derived and measured functions is $47.98 \%$. In contrast with it, the derived Mooney Rivlin models have very agreement with the experimental results, even the 2 parameters model
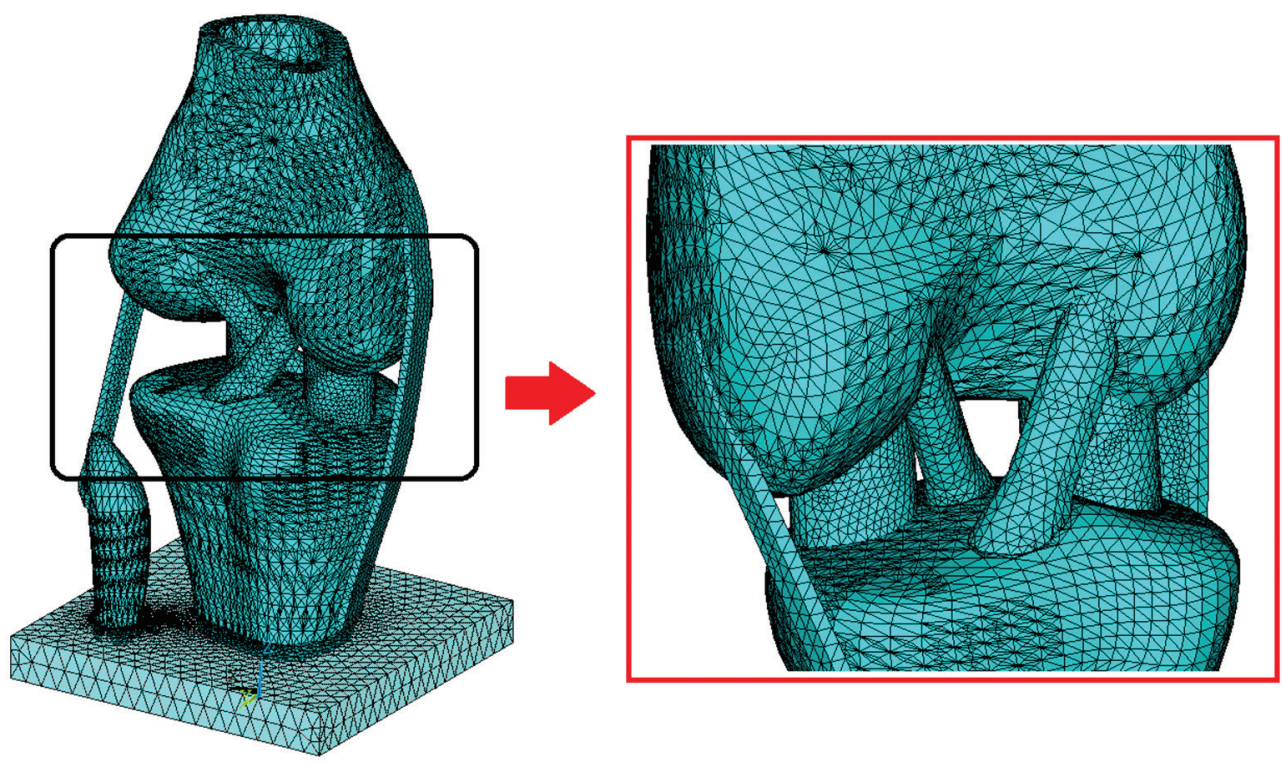

Figure 4. Finite element model and mesh of the knee joint 
show $99.85 \%$ coincidence, thus this family of hyperelastic models is absolutely adequate for the modelling of soft tissues.

\subsection{Spatial numerical modelling of the knee joint}

For the numerical simulations a three dimensional model of the knee joint has been developed in Ansys. It contains the three major bones (femur, tibia and fibula), the four major ligaments (cruciate and collateral ligaments) and the two menisci. The best way to prepare the geometric model is to use the results of MRI/CT scans, but now due to the lack of such resources it was not the case. For the modelling of the bones a CAD model is used, ${ }^{12}$ while for the ligaments and menisci the geometry is created in the program based on the available measurement results on these constituents. ${ }^{13-17}$ The numerical model containing solid finite elements can be seen on Figure 4. A rigid plate connects the tibia and fibula in order to hinder the relative motions between them, practically it is functioning as a spacer.

Due to the numerous constituents which material behaviour is significantly diverse, two types of material models with different material parameters are applied in the model, linear elastic for the menisci and bones and 5 parameters Mooney-Rivlin hyperelastic for the ligaments. The applied values of the material parameters are based on the work of Pena et al. ${ }^{17}$ The model of the knee joint is subjected to various knee motions in order to investigate the behavior of the collateral and cruciate ligaments. These loading cases involve $5 \mathrm{~mm}$ anterior and posterior tibial translation, $5^{\circ}$ tibial valgus and varus rotation and $5^{\circ}$ internal and external rotation (Figure 5).

Due to the volume elements, the rotations were defined by equivalent translations on the tibia and fibula. In case of valgus rotation, the

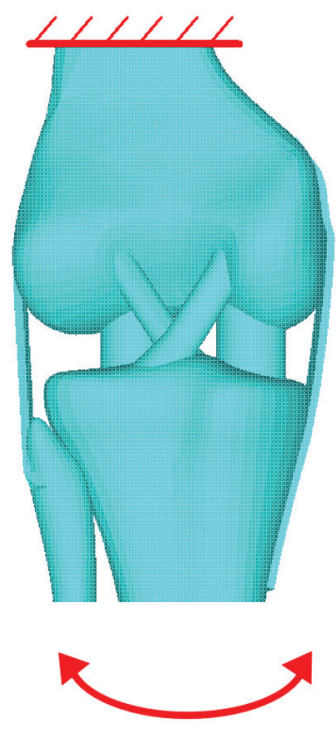

a)

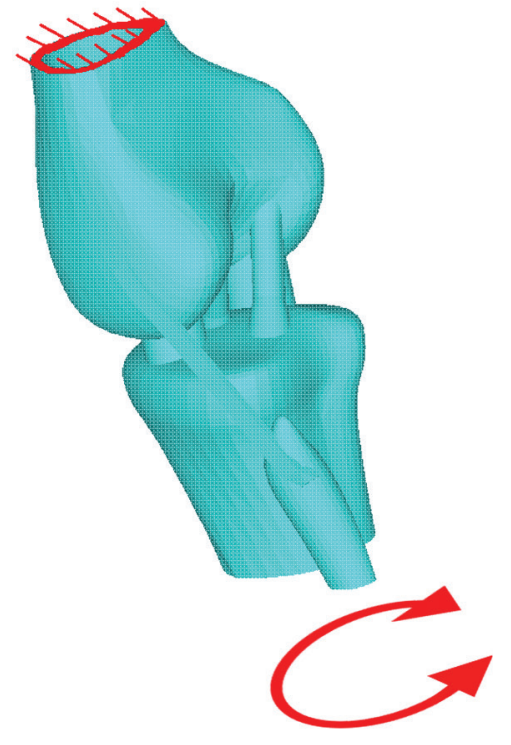

b)

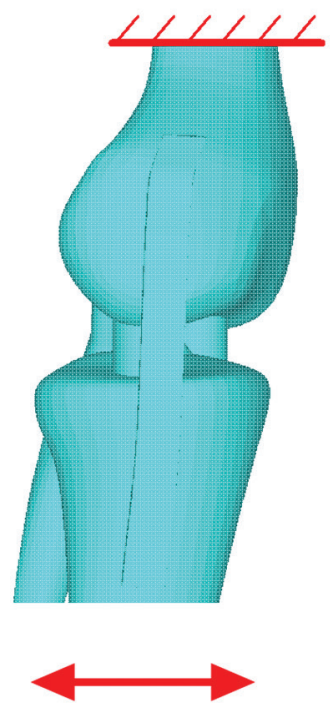

c)

Figure 5. The investigated knee motions during the simulations: a) valgus/varus rotation; b) internal/external rotation; c) anterior/posterior translation 
MCL and the PCL carries the majority of the external load, furthermore a "buckling" effect appeared in the LCL. By the comparison of the maximal von Mises stresses practically the MCL is the only stabilizing ligament in this case (the difference is very large between the participation of the two ligaments), it is about $25 \mathrm{MPa}$. At varus rotation, the other two ligaments bear the load, the ACL and LCL, but the portions of the carried load are significantly closer to each other: about $12 \mathrm{MPa}$ in the ACL and $28 \mathrm{MPa}$ in the LCL. At varus valgus/rotation practically the collateral ligaments responsible for the stability of the joint. At internal rotation, all ligaments take part in the load-bearing, but mostly the collateral ligaments, the maximum stress was the same in the LCL and MCL, about 2.5 MPa. In case of external rotation higher stresses appeared, furthermore the maximal stress in the collateral ligaments are not equal, in the LCL it is higher (about $4 \mathrm{MPa}$ ) while in the MCL it is $3 \mathrm{MPa}$. In case of internal/external rotation, all ligaments contribute to the stability of the joint, and the developing stresses are one order lower comparing to the varus/valgus rotation. At the anterior tibial translation the majority of the load is carried by the cruciate ligaments, especially by the ACL, in which the maximal stress is about $42 \mathrm{MPa}$ while in the PCL it is $20 \mathrm{MPa}$. In case of posterior tibial translation, the carried portions of the load by the cruciate ligaments are almost the same, in the ACL the maximal stress is about $38 \mathrm{MPa}$ and in the PCL it is $43 \mathrm{MPa}$.

\section{Discussion and conclusions}

The purpose of this work is to investigate the structure, mechanical behaviour and numerical modelling techniques/possibilities of ligaments. Due to the frequent injuries, it is very important to investigate them in details from both medical and mechanical point of view, this topic contains a lot of research possibili- ties. The application of artificial ligaments is also a continuously developing area due to its necessity, but up to now it cannot provide the adequate level of safety or guarantee the longterm success.

The correct definition of the material behaviour is essential to the adequate numerical simulations, currently the hyperelastic models are the most popular choice for this purpose. They can accurately describe the mechanical response with the help of the strain energy density function and have a very good finite element implantation capability. On the basis of laboratory experiments, different hyperelastic models (Neo-Hooke, Mooney Rivlin) are parameterized by the utilization of the results of uniaxial tensile tests. Although four different Mooney-Rivlin model are created, the two parameters version is practically enough to describe precisely the mechanical behaviour.

For numerical simulations a spatial finite element model has been developed in Ansys using volume elements, containing the major bones, ligaments and menisci of the knee joint. The hyperelastic material models applied at the ligaments are based on real experiments, furthermore the bones and the menisci are assumed to have linearly elastic behaviour. This model is used to investigate different knee motions, namely $5^{\circ}$ valgus and varus rotation, $5^{\circ}$ internal and external rotation and $5 \mathrm{~mm}$ anterior and posterior tibial translations. Due to the volume elements, the rotations were defined by equivalent translations. At rotational motions the collateral ligaments carry the majority of the load, the higher stresses developing in the case of varus/valgus rotations. For translational motions the cruciate ligaments are the main stabilizing constituents of the knee joint, the developing stresses are similar for the anterior and posterior motions. 


\section{REFERENCES}

1. Gunther T. A térdízület biomechanikájának változása az unikompartmentális térdprotézis beültetése során [dissertation]. Budapest (HUN): Semmelweis Univ., 2001.

2. Kastelic J, Galeski A, Baer E. Multicomposite structure of tendon. Connective Tissue Research $1978 ; 6(1): 11-23$.

3. Vita, $R D$. Structural constitutive model for knee Ligaments [dissertation]. Pennsylvania (USA): University of Pittsburgh, 2005.

4. Holzapfel GA. Biomechanics of soft tissue. In: Lemaitre J, editor. Handbook of materials behaviour models 1. New York: Academic Press; 2000. p. 1057-75.

5. Haghpanahi $M$, Jalayer $F$ Three-dimensional finite element analysis of the human ACL. Proceedings of the 1st WSEAS International Conference; 2008 Aug 20-22; Rhodes, Greece.

6. Weiss JA, Gardiner JC. Computational modeling of ligament mechanics. Critical Reviews in Biomedical Engineering 2001;29(3):303-71.

7. Xie F, Yang L, Guo L, Wang Z, Dai G. A Study on construction three-dimensional nonlinear finite element model and stress distribution analysis of anterior cruciate ligament. Journal of Biomechanical Engineering 2009;(13112):121007.

8. Untaroiu C, Darvish K, Crandall J, Bing D, Wang JT. Characterization of the lower limb soft tissues in pedestration finite element models. Proceedings of the 19th International Technical Conference on the Enhanced Safety of Vehicles; 2005 June 6-9; Washington DC, USA.

9. Weiss JA, Gardiner JC. GSubject-specific Finite element analysis of the human medial collateral ligament during valgus knee loading. Journal of Orthopedic Research 2003;21(6):1098-106.

10. Yan-lin Z, You W, Hai-peng W, Ke R, Le X. Stress changes of lateral collateral ligament at different knee flexion with or without displaced movements: A 3-dimensional finite element analysis. Chinese Journal of Traumatology 2011;14(2):7983.

11. Odgen $R W$. Background on nonlinear elasticity. In: Lemaitre J, editor. Handbook of materials behaviour models 1. New York: Academic Press; 2000. p. 75-84.

12. Human Skeleton. [cited 2013 Jan 2]. Available from: URL: https:/grabcad.com/library/ human-skeleton-used-for-a-parametric-gaitstudy-1.

13. Lipps DB, Oh Y, Wojtys EM, Ashton-Miller JA. ACL cross-sectional area and medial tibial platea geometry provide insights on the gender difference in the peak ACL strain. Annual Meeting of the American Society of Biomechanics; 2011 Aug 10-13; Long Beach, USA. Available from: URL: http://www.asbweb.org/conferences/2011/pdf/249.pdf.

14. Hara K, Mochizuki T, Sekiya I, Yamaguchi K, Akita $K$, Muneta T. Anatomy of normal human anterior cruciate ligament attachments evaluated by divided small bundles. The American Journal of Sports Medicine 2009;(3712):2386-91.

15. Keats TE, Christopher S. Atlas of radiologic measurement. 7th ed. St. Louis: Mosby; 2001.

16. Lai MH, Chang ST, Chou YC, Chang CH, Wang TY, Huang HC, et al. Real-time ultrasonographic characteristics of the lateral collateral ligament of the knee in cross-leg position with varus stress maneuver. Journal of Medical Science 2011;31(4):153-9.

17. Pena E, Calvo B, Martınez M, Doblare M. A three-dimensional finite element analysis of the combined behavior of ligaments and menisci in the healthy human knee joint. Journal of Biomechanics 2006;39(9):1686-701.

\section{Ferenc Szakály}

Department of Structural Mechanics, Budapest University of Technology and Economics H-1111 Budapest, Múegyetem rkp. 3. K building, KM 63.

Tel.: (+36) 1 463-2201 